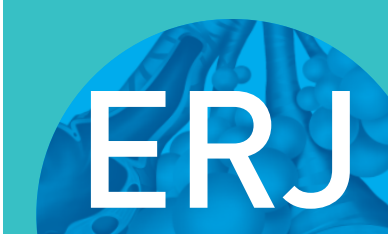

open research

\title{
Regional distribution of high-attenuation areas on chest computed tomography in the Multi-Ethnic Study of Atherosclerosis
}

\author{
Bina Choi ${ }^{1}$, Steven M. Kawut ${ }^{2}$, Ganesh Raghu ${ }^{3}$, Eric Hoffman ${ }^{4}$, Russell Tracy ${ }^{5}$, \\ Purnema Madahar ${ }^{1}$, Elana J. Bernstein (1) ${ }^{1}$, R. Graham Barr ${ }^{1}$, David J. Lederer (1) ${ }^{1}$ \\ and Anna Podolanczuk (i) ${ }^{1}$
}

Affiliations: ${ }^{1}$ Columbia University Medical Center, New York, NY, USA. ${ }^{2}$ University of Pennsylvania School of Medicine, Philadelphia, PA, USA. ${ }^{3}$ University of Washington Medical Center, Seattle, WA, USA. ${ }^{4}$ University of lowa Carver College of Medicine, lowa City, IA, USA. ${ }^{5}$ University of Vermont, Burlington, VT, USA.

Correspondence: Anna Podolanczuk, Columbia University Medical Center, 177 Fort Washington, New York, NY 10032, USA. E-mail: ajp2158Rcumc.columbia.edu

ABSTRACT High-attenuation areas (HAA) are a computed tomography-based quantitative measure of subclinical interstitial lung disease (ILD). We aimed to validate HAA in lung regions that are less subject to artefacts, such as extravascular lung water or dependent atelectasis. We examined the associations of HAA within six lung regions (basilar, non-basilar, peel, core, basilar peel, basilar core) with serum biomarkers of lung remodelling, forced vital capacity (FVC), visually-assessed interstitial lung abnormalities (ILA), and all-cause and ILD-specific mortality.

We performed cross-sectional and longitudinal analyses of participants in the Multi-Ethnic Study of Atherosclerosis, a prospective cohort of 6814 adults aged 45-84 years without known cardiovascular disease who underwent cardiac computed tomography.

Median regional HAA ranged from $3.8 \%$ in the peel to $4.8 \%$ in the basilar core. Doubling of regional HAA was associated with greater serum matrix metalloproteinase-7 (range 3.8\% to $10.3 \%$; $\mathrm{p} \leqslant 0.01$ ), higher odds of ILA (OR 1.42 to 2.20; $\mathrm{p} \leqslant 0.03$ ), and a higher risk of all-cause mortality (hazard ratio 1.20 to 1.47; $\mathrm{p} \leqslant 0.001$ ). Doubling of regional HAA was associated with greater serum interleukin-6 (4.9\% to $10.3 \%$; $\mathrm{p} \leqslant 0.005$ ) and higher risk of ILD-specific mortality (hazard ratio 3.30 to $3.98 ; \mathrm{p}<0.001$ ), except in the basilar core. Doubling of regional HAA was associated with lower FVC in the non-basilar, core and basilar core $(113 \mathrm{~mL}$ to $186 \mathrm{~mL}$; $\mathrm{p}<0.001)$.

Associations of HAA with lung remodelling biomarkers, ILA risk and all-cause mortality were consistent across all regions of the lung, including dependent areas where atelectasis may be present. These findings support the validity of HAA as a measure of pathologic subclinical ILD.

@ERSpublications

Even when found in small regions of the lungs, high-attenuation areas, a CT-based quantitative measure of subclinical ILD, are associated with biomarkers of lung remodelling, risk of interstitial lung abnormalities and all-cause mortality http://bit.ly/36psfin

Cite this article as: Choi B, Kawut SM, Raghu G, et al. Regional distribution of high-attenuation areas on chest computed tomography in the Multi-Ethnic Study of Atherosclerosis. ERJ Open Res 2020; 6: 00115-2019 [https://doi.org/10.1183/23120541.00115-2019].

This article has supplementary material available from openres.ersjournals.com

Received: 9 May 2019 | Accepted after revision: 4 Dec 2019

Copyright $\odot$ ERS 2020. This article is open access and distributed under the terms of the Creative Commons Attribution Non-Commercial Licence 4.0. 


\section{Introduction}

The interstitial lung diseases (ILD) are a heterogeneous group of disorders characterised by alveolar injury, inflammation and extracellular matrix remodelling [1,2]. Adults with subclinical ILD have radiological, histopathological and functional changes that precede the development of clinically evident ILD [3]. Studies of subclinical ILD may lead to improved detective, preventative and treatment strategies for ILD.

Subclinical ILD can be identified using computer-based, automated approaches to detect early interstitial changes in adults without symptoms or a prior diagnosis of ILD [4-7]. High-attenuation areas (HAA) are one example of a validated computer tomography (CT)-derived, quantitative phenotype of subclinical ILD [8-10]. We have previously shown that HAA is independently associated with inflammatory biomarkers, reduced lung function, visually identified interstitial lung abnormalities (ILA) and higher risk of all-cause and ILD-specific mortality $[8,11]$. We have used HAA and other measures to identify novel risk factors for ILD, including obstructive sleep apnoea and high-density lipoprotein cholesterol $[12,13]$.

Given the lower specificity of HAA as a measure of early lung fibrosis compared to other automated approaches, it has been suggested that HAA may be an artefact caused by pulmonary oedema or dependent atelectasis [14-16]. Therefore, in this study, we aimed to validate HAA in lung regions that are less subject to these artefacts. We examined associations of HAA within six lung regions (basilar, non-basilar, peel, core, basilar peel and basilar core) with matrix metalloproteinase (MMP)-7, interleukin (IL)-6, surfactant protein (SP)-A, forced vital capacity (FVC), ILA and all-cause and ILD-specific mortality. MMP-7 and SP-A were chosen as biomarkers of lung remodelling and injury and IL-6 was chosen as a biomarker of systemic inflammation [8, 17-23]. We hypothesised that HAA in the non-basilar regions would be more strongly associated with biomarkers of injury and remodelling, lower FVC, higher odds of ILA, and mortality. Secondarily, we aimed to examine whether HAA in the outer regions of the lung are more strongly associated with ILA.

\section{Methods}

\section{Study design and participants}

We performed cross-sectional and longitudinal analyses of participants enrolled in the Multi-Ethnic Study of Atherosclerosis (MESA). MESA is a prospective cohort of 6814 adults aged 45-84 year without known cardiovascular disease at baseline, drawn from six communities in the USA. Enrollment criteria have been described previously; there were no selection criteria based on lung disease, respiratory symptoms or smoking history [24]. At the baseline visit (years 2000-2002), participants underwent cardiac CT scans and received a blood draw for measurements of the biomarkers MMP-7, IL-6 and SP-A. Participants were followed longitudinally with contact every 9-12 months and with serial follow-up visits. At the examination 3 and 4 follow-up visits (years 2004-2007), a random sample of participants underwent spirometry. At the examination 5 follow-up visit (years 2010-2012), a subset of participants underwent full-lung CT scans that were then assessed for ILA. The National Death Index (NDI) was reviewed to assess mortality. Death due to ILD was adjudicated as previously described [11].

\section{Regional HAA}

HAA was assessed on full inspiratory cardiac CT scans obtained at the baseline visit. The scans were performed twice and the scan with the lowest density was used to assure the best inspiratory effort. HAA was defined as the percentage of voxels with attenuation between -600 and -250 Hounsfield units, as previously described [8]. Regional HAA was calculated for each of the lung regions described below. All participants received complete cardiac CT scans but regional HAA was available for 6671 to 6810 participants ( $>97 \%$ ); the missing data were removed for poor quality, incomplete image or excessive artefact.

To define the lung regions, CT scans were divided into three lung zones. These three zones on cardiac CT scans do not correspond with upper, middle (or lingula) and lower lobes of the lungs. Rather, they are the upper, middle and lower thirds of the craniocaudal extent of the cardiac CT scan. Previous studies have shown good correlation between the caudal eighth of cardiac CTs and the caudal third of full-lung CTs for percentage emphysema [25]. The basilar (dependent) region included the caudal third of the imaged lung. The non-basilar region included the remaining two-thirds. The peel region included the outer $20 \mathrm{~mm}$ of the lung, and the core region included the remaining inner axial portions. With these definitions, we analysed six distinct axial and craniocaudal lung regions: basilar, non-basilar, peel, core, basilar peel and basilar core (figure 1).

\section{Biomarkers, spirometry and ILA}

The biomarkers MMP-7, IL-6 and SP-A were measured from participants' serum obtained at examination 1 using quantitative sandwich ELISA assays, as previously described [8]. 


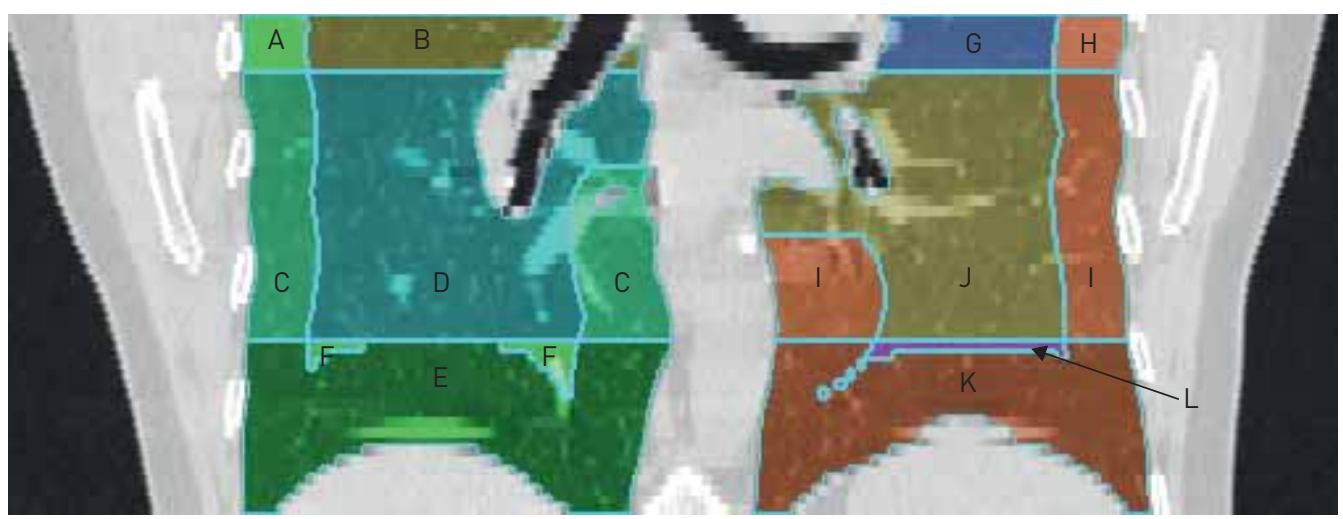

FIGURE 1 Multi-Ethnic Study of Atherosclerosis (MESA) cardiac computed tomography scan with superimposed lines to delineate examined lung regions. In this representative scan, the basilar region includes sections $E, F, K$, L. The non-basilar region includes sections $A, B, C, D, G, H, I, J$. The peel includes sections $A, C, E, H, I, K$. The core includes sections $B, D, F, G, J, L$. The basilar peel includes sections $E, K$. The basilar core includes sections F, L.

Spirometry was performed at examination 3 according to the American Thoracic Society/European Respiratory Society guidelines, as previously described [26].

ILA was assessed on full-lung CT scans performed at examination 5 (approximately 10 years after the baseline cardiac CTs), as previously described $[27,28]$. The CTs were read by one of five trained readers, who scored the presence of ILA if the images contained ground-glass opacity, reticular pattern, nonemphysematous cysts, honeycombing, traction bronchiectasis or centrilobular nodules in at least $5 \%$ of nondependent portions of the lung $[4,6,7]$. Indeterminate scans were excluded from analyses.

\section{Statistical analysis}

We used generalised linear models to examine the associations of regional HAA with MMP-7, IL-6, SP-A, FVC and ILA. Regional HAA, MMP-7, IL-6 and SP-A were binary log-transformed, and we report effect estimates per doubling of regional HAA. We used Cox hazards models to examine the associations of regional HAA with time to death and ILD death. All models were adjusted for age, sex, race, BMI, height, waist circumference, mAs (milliampere-seconds), regional volume of imaged lung, smoking status, pack-years, regional percentage emphysema, educational attainment, glomerular filtration rate and study site. Due to low event rates, models for ILD death were adjusted for a generalised propensity score (GPS), as previously described [11]. This included the above covariates, as well as alcohol use, total intentional exercise (metabolic equivalent $\mathrm{min} \cdot \mathrm{wk}^{-1}$ ), coronary artery calcium, diabetes medication use, insulin use, fasting glucose, hypertension, antihypertensive medication use, systolic and diastolic blood pressures, cholesterol medication use, total and high-density lipoprotein cholesterol levels, C-reactive protein, D-dimer and cancer history. We also performed analyses stratified by BMI category and smoking status. We used likelihood ratio tests to evaluate for effect modification by BMI and smoking status. All analyses were performed using Stata version 14.2 (StataCorp, College Station, TX, USA).

\section{Results}

The baseline characteristics of all participants are shown in table 1. Participants had a mean age of 62, $53 \%$ were female, $13 \%$ were current smokers and $37 \%$ were former smokers. Participants had a mean BMI of $28 \mathrm{~kg} \cdot \mathrm{m}^{-2}$; $39 \%$ were overweight (BMI $25-30 \mathrm{~kg} \cdot \mathrm{m}^{-2}$ ) and $32 \%$ were obese (BMI $>30 \mathrm{~kg} \cdot \mathrm{m}^{-2}$ ). The median (IQR) regional HAA ranged from $3.8 \%(3.1-5.0 \%)$ in the peel to $4.8 \%(3.8-6.6 \%)$ in the basilar core region (figure 2 and Table S1). Correlation coefficients amongst different areas ranged from 0.68 to 0.90 (Table S2).

\section{Biomarkers}

Greater regional HAA was associated with higher serum MMP-7 and IL-6 levels within each lung region and the magnitude of these associations was smallest in the basilar core region (table 2). For each doubling of regional HAA, there was an associated increment in MMP-7 ranging from 3.8\% (95\% CI 0.8-7.0\%) in the basilar core to $10.3 \%$ (95\% CI $4.7-16.3 \%)$ in the core regions, and an associated increment in IL-6 ranging from $1.5 \%(95 \% \mathrm{CI}-0.9-4.0 \%)$ in the basilar core to $10.3 \%$ (95\% CI $6.5-14.2 \%)$ in the non-basilar regions. There were no significant associations of regional HAA with SP-A, except for the core region, where each doubling of HAA was associated with an $8.7 \%$ increment (95\% CI $0.2-17.8 \%$ ) in SP-A. 


\section{TABLE 1 Baseline characteristics of Multi-Ethnic Study of Atherosclerosis (MESA) participants}

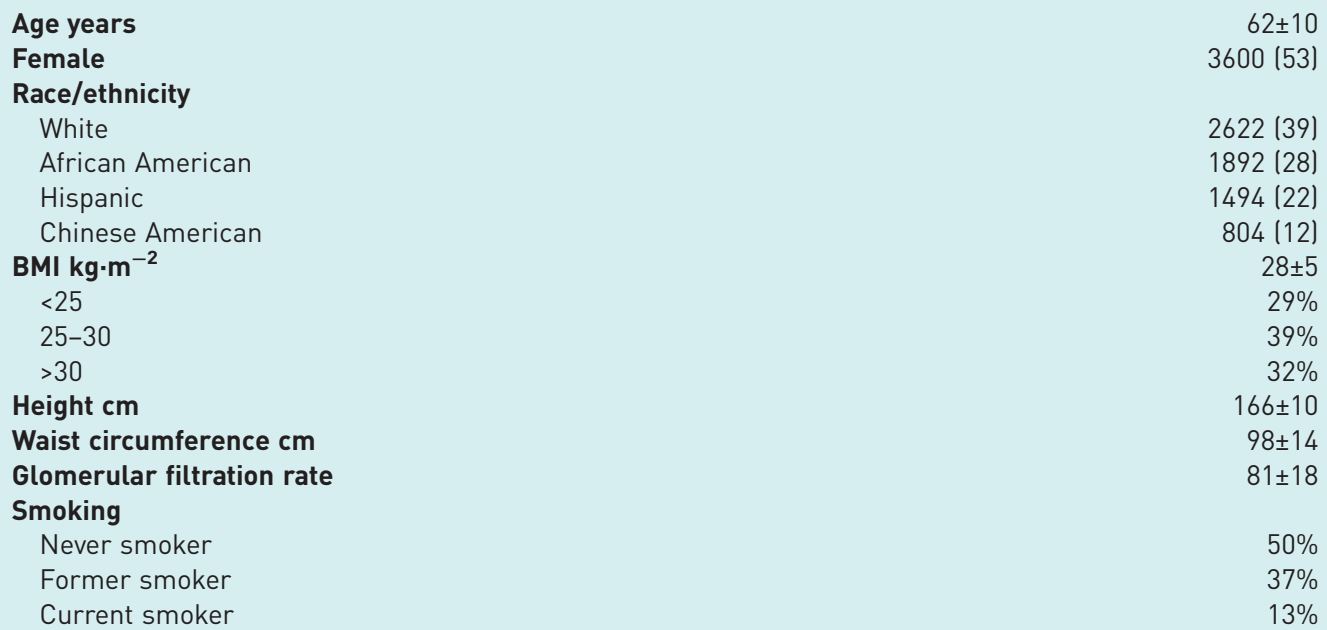

Height $\mathrm{cm}$

Waist circumference $\mathrm{cm}$

Glomerular filtration rate

\section{Smoking}

Never smoker

Former smoker

Current smoker

Data are presented as mean \pm SD or $n(\%)$, unless otherwise stated. All parameters collected at MESA baseline visit in years 2000-2002. BMI: body mass index.

There was evidence of effect modification by smoking status on the association between regional HAA and MMP-7 ( $\mathrm{p}$ for interaction $<0.005$ for all lung regions), with stronger associations within each lung region among ever-smokers compared to never smokers (Table S3). There was evidence of effect modification ( $\mathrm{p}$ for interaction $<0.05$ ) by BMI on the association between regional HAA and MMP-7 in the

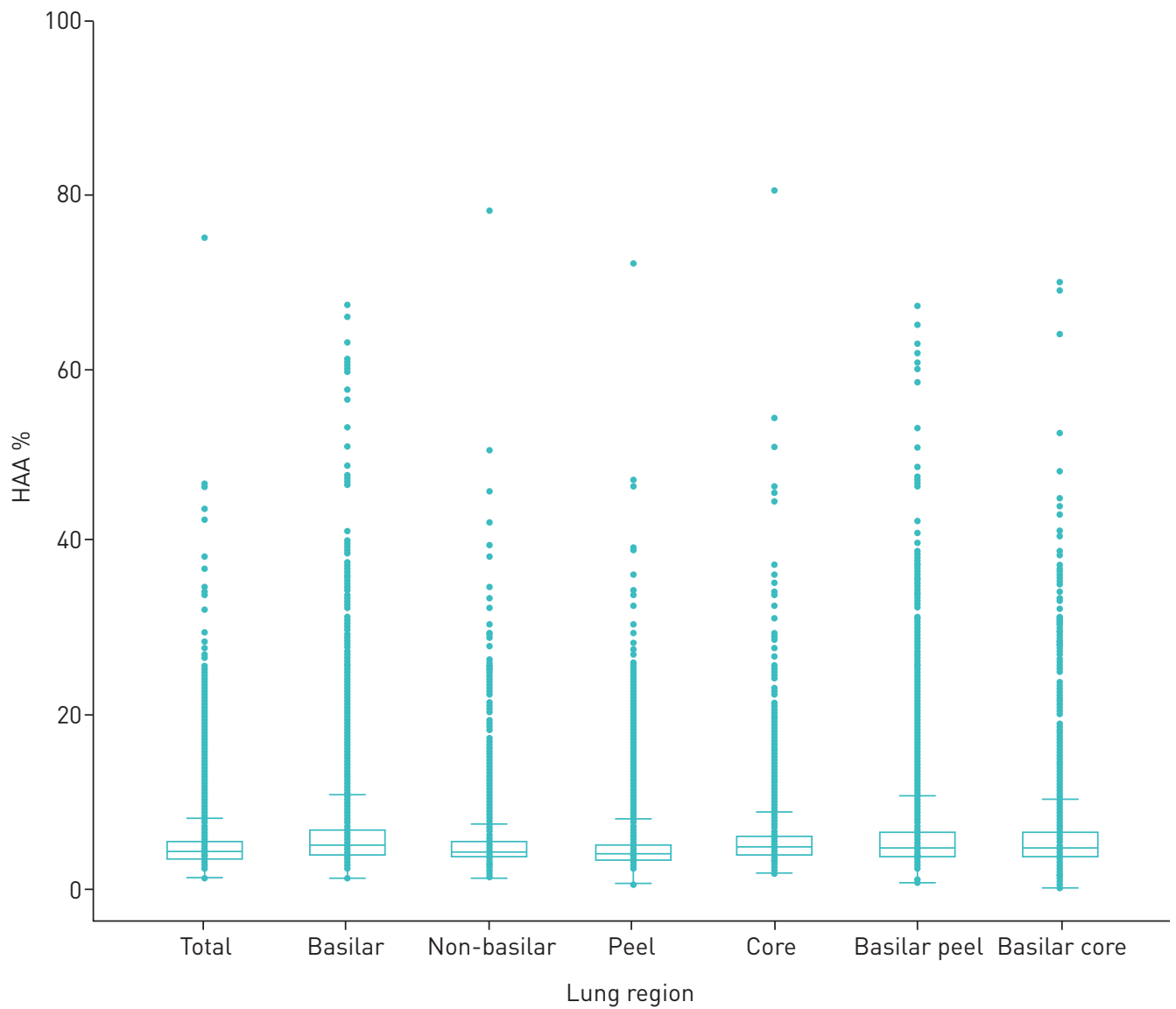

FIGURE 2 Baseline regional high-attenuation areas (HAA). 
TABLE 2 Associations of biomarkers, forced vital capacity, ILA, and all-cause and ILD-specific mortality with HAA in examined lung regions

\begin{tabular}{|c|c|c|c|c|c|c|c|c|c|c|c|c|}
\hline & \multicolumn{2}{|l|}{ Basilar } & \multicolumn{2}{|c|}{ Non-basilar } & \multicolumn{2}{|l|}{ Peel } & \multicolumn{2}{|l|}{ Core } & \multicolumn{2}{|c|}{ Basilar peel } & \multicolumn{2}{|c|}{ Basilar core } \\
\hline & $\begin{array}{c}\text { Change per } \\
\text { doubling HAA \% }\end{array}$ & $\mathrm{p}$-value & $\begin{array}{c}\text { Change per } \\
\text { doubling HAA \% }\end{array}$ & $p$-value & $\begin{array}{c}\text { Change per } \\
\text { doubling HAA \% }\end{array}$ & $\mathrm{p}$-value & $\begin{array}{c}\text { Change per } \\
\text { doubling HAA \% }\end{array}$ & $\mathrm{p}$-value & $\begin{array}{c}\text { Change per } \\
\text { doubling HAA \% }\end{array}$ & $\mathrm{p}$-value & $\begin{array}{c}\text { Change per } \\
\text { doubling HAA \% }\end{array}$ & p-value \\
\hline MMP-7 & $6.6(2.6-10.8)$ & 0.001 & $6.4(2.5-10.4)$ & 0.001 & $5.9(2.2-9.8)$ & 0.002 & $10.3(4.7-16.3)$ & $<0.001$ & $5.8(2.2-9.6)$ & 0.002 & $3.8(0.8-7.0)$ & 0.01 \\
\hline IL-6 & $4.9(1.5-8.4)$ & 0.005 & $10.3(6.5-14.2)$ & $<0.001$ & $7.3(4.0-10.6)$ & $<0.001$ & $9.1(4.0-14.4)$ & $<0.001$ & $5.5(2.4-8.6)$ & $<0.001$ & $1.5(-0.9-4.0)$ & 0.220 \\
\hline SP-A & $\begin{array}{c}4.9(-1.1-11.3) \\
\text { Change per } \\
\text { doubling HAA } \mathrm{mL}\end{array}$ & $\begin{array}{c}0.113 \\
\text { p-value }\end{array}$ & $\begin{array}{c}2.7(-3.0-8.8) \\
\text { Change per } \\
\text { doubling HAA mL }\end{array}$ & $\begin{array}{c}0.362 \\
\text { p-value }\end{array}$ & $\begin{array}{c}5.2[-0.5-11.1] \\
\text { Change per } \\
\text { doubling HAA } \mathrm{mL}\end{array}$ & $\begin{array}{c}0.073 \\
\mathbf{p} \text {-value }\end{array}$ & $\begin{array}{c}8.7(0.2-17.8) \\
\text { Change per } \\
\text { doubling HAA } \mathrm{mL}\end{array}$ & $\begin{array}{c}0.04 \\
\text { p-value }\end{array}$ & $\begin{array}{c}4.2(-1.3-10.0) \\
\text { Change per } \\
\text { doubling HAA } \mathrm{mL}\end{array}$ & $\begin{array}{c}0.140 \\
\text { p-value }\end{array}$ & $\begin{array}{c}-0.1(-4.7-4.8) \\
\text { Change per } \\
\text { doubling HAA } \mathrm{mL}\end{array}$ & $\begin{array}{c}0.973 \\
\text { p-value }\end{array}$ \\
\hline FVC & $\begin{array}{c}-28(-57-0) \\
\text { OR per doubling } \\
\text { HAA }\end{array}$ & $\begin{array}{c}0.051 \\
\text { p-value }\end{array}$ & $\begin{array}{c}-113(-151--75) \\
\text { OR per doubling } \\
\text { HAA }\end{array}$ & $\begin{array}{l}<0.001 \\
\mathbf{p} \text {-value }\end{array}$ & $\begin{array}{c}-26(-58-5) \\
\text { OR per doubling } \\
\text { HAA }\end{array}$ & $\begin{array}{c}0.099 \\
\mathbf{p} \text {-value }\end{array}$ & $\begin{array}{c}-186(-226--145) \\
\text { OR per doubling } \\
\text { HAA }\end{array}$ & $\begin{array}{l}<0.001 \\
\mathbf{p} \text {-value }\end{array}$ & $\begin{array}{c}-15(-42-12) \\
\text { OR per doubling } \\
\text { HAA }\end{array}$ & $\begin{array}{c}0.226 \\
\mathbf{p} \text {-value }\end{array}$ & $\begin{array}{c}-115(-142--88) \\
\text { OR per doubling } \\
\text { HAA }\end{array}$ & $\begin{array}{l}<0.001 \\
\mathbf{p} \text {-value }\end{array}$ \\
\hline ILA & $\begin{array}{c}2.20(1.66-2.90) \\
\text { Hazard ratio per } \\
\text { doubling HAA }\end{array}$ & $\begin{array}{l}<0.001 \\
\mathbf{p} \text { Value }\end{array}$ & $\begin{array}{c}1.54(1.17-2.02) \\
\text { Hazard ratio per } \\
\text { doubling HAA }\end{array}$ & $\begin{array}{l}0.002 \\
\text { p Value }\end{array}$ & $\begin{array}{c}1.79(1.40-2.30) \\
\text { Hazard ratio per } \\
\text { doubling HAA }\end{array}$ & $\begin{array}{l}<0.001 \\
\mathbf{p} \text { Value }\end{array}$ & $\begin{array}{c}1.52(1.04-2.24) \\
\text { Hazard ratio per } \\
\text { doubling HAA }\end{array}$ & $\begin{array}{l}0.03 \\
\mathbf{p} \text { Value }\end{array}$ & $\begin{array}{c}1.87(1.45-2.39) \\
\text { Hazard ratio per } \\
\text { doubling HAA }\end{array}$ & $\begin{array}{l}<0.001 \\
\mathbf{p} \text { Value }\end{array}$ & $\begin{array}{c}1.42(1.14-1.76) \\
\text { Hazard ratio per } \\
\text { doubling HAA }\end{array}$ & $\begin{array}{l}0.001 \\
\text { p Value }\end{array}$ \\
\hline $\begin{array}{l}\text { All-cause } \\
\text { mortality }\end{array}$ & $1.25(1.10-1.42)$ & $<0.001$ & $1.47(1.29-1.66)$ & $<0.001$ & $1.41(1.26-1.58)$ & $<0.001$ & $1.35(1.13-1.62)$ & 0.001 & $1.21(1.08-1.36)$ & 0.001 & $1.20(1.09-1.32)$ & $<0.001$ \\
\hline ILD mortality" & $3.98(2.10-7.55)$ & $<0.001$ & $3.44(2.13-5.56)$ & $<0.001$ & $3.94(2.36-6.56)$ & $<0.001$ & $3.30(1.83-5.93)$ & $<0.001$ & $3.89(2.08-7.29)$ & $<0.001$ & $1.83(0.97-3.46)$ & 0.061 \\
\hline
\end{tabular}

Data are presented as mean (95\% CI). All analyses adjusted for age, sex, race/ethnicity, body mass index (BMI), waist circumference, smoking status, pack-years, educational attainment, renal function, total volume of imaged lung (not in FVC analysis), percentage emphysema, mA dose and study site. HAA: high attenuation areas; MMP-7: matrix metalloproteinase-7; IL6: interleukin-6; SP-A: surfactant protein-A; FVC: forced vital capacity; ILA: interstitial lung abnormalities. \#: Adjusted for generalised propensity score, which includes age, sex, race/ ethnicity, BMI, waist circumference, smoking status, pack-years, educational attainment, renal function, total volume of imaged lung, percentage emphysema, mA dose, study site, alcohol use, total intentional exercise (metabolic equivalent min.wk ${ }^{-1}$ ), coronary artery calcium, diabetes medication use, insulin use, fasting glucose, hypertension, antihypertensive medication use, systolic and diastolic blood pressures, cholesterol medication use, total and high-density lipoprotein cholesterol levels, C-reactive protein, D-dimer and cancer history. 
non-basilar, core and basilar core regions. Associations were strongest in the overweight group (BMI $25-30 \mathrm{~kg} \cdot \mathrm{m}^{-2}$ ) (Table S4). There was no evidence of effect modification by smoking or BMI on the association between regional HAA and IL- 6 or SP-A in any of the lung regions ( $\mathrm{p}$ for interaction $>0.06$, data not shown).

\section{Forced vital capacity}

Higher regional HAA was associated with lower FVC in all lung regions, but this association was not statistically significant in the basilar, peel and basilar peel regions (table 2). The association between HAA and lower FVC was of the greatest magnitude in the core region, where each doubling of HAA was associated with a $186 \mathrm{~mL}(95 \%$ CI $145-226 \mathrm{~mL})$ decrement in FVC.

There was evidence of effect modification by smoking status on the association between regional HAA and FVC, with the association strongest among ever-smokers (Table S3). The effect of smoking status was greatest in the core and least in the basilar regions. There was no evidence of effect modification by BMI on the association between regional HAA and FVC in any of the lung regions ( $p$ for interaction $>0.10$; Table S4).

\section{Interstitial lung abnormalities}

Greater regional HAA in all lung regions was associated with higher odds of overall ILA (figure 3). Doubling of regional HAA in each region was associated with higher odds of ILA, with OR ranging from 1.42 (CI 1.14-1.76) in the basilar core to 2.20 (CI 1.66-2.90) in the basilar regions (table 2). There was no evidence of effect modification by smoking or BMI on the association between regional HAA and ILA in any of the lung regions ( $\mathrm{p}$ for interaction $>0.10$; data not shown).

\section{Mortality}

Greater regional HAA in all lung regions was associated with increased mortality (figure 4). Doubling of regional HAA in each region was associated with higher hazard ratio of all-cause mortality ranging from 1.20 (CI 1.09-1.32) in the basilar core to 1.47 (CI 1.29-1.66) in the non-basilar regions (table 2). There was no evidence of effect modification by smoking or BMI on the association between regional HAA and mortality in any of the lung regions ( $\mathrm{p}$ for interaction $>0.682$, data not shown).

There were 20 ILD deaths in the cohort. Greater regional HAA was associated with increased ILD-specific mortality. Doubling of regional HAA in each region except the basilar core was associated with a higher hazard ratio of ILD death ranging from 3.30 (CI 1.83-5.93) in the core to 3.98 (CI 2.10-7.55) in the basilar regions (table 2).

\section{Discussion}

High attenuation areas (HAA), the number of lung voxels between -600 and -250 Hounsfield units, were previously validated as a quantitative measure of subclinical ILD on chest CT. In the current study, we found that regional HAA calculated from portions of the cardiac CTs, divided into regions, was consistently associated with biomarkers of lung injury and inflammation, reduced lung function, visually-assessed interstitial lung abnormalities (ILA), and higher risk of all-cause and ILD mortality.

We had hypothesised that HAA in the non-basilar region would be less subject to artefacts such as pulmonary oedema or dependent atelectasis and, therefore, would be more strongly associated with our outcomes. Interestingly, and somewhat contrary to our hypothesis, we found similar associations when comparing HAA in the basilar (dependent) regions to the non-basilar regions and peel compared with core

FIGURE 3 Odds ratios (OR; with 95\% confidence intervals) of interstitial lung abnormalities (ILA) per doubling of high-attenuation areas (HAA).

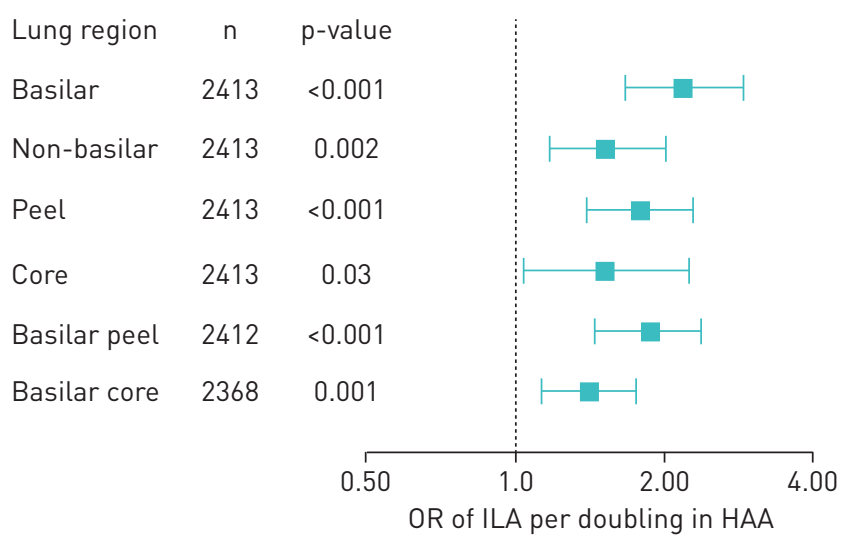




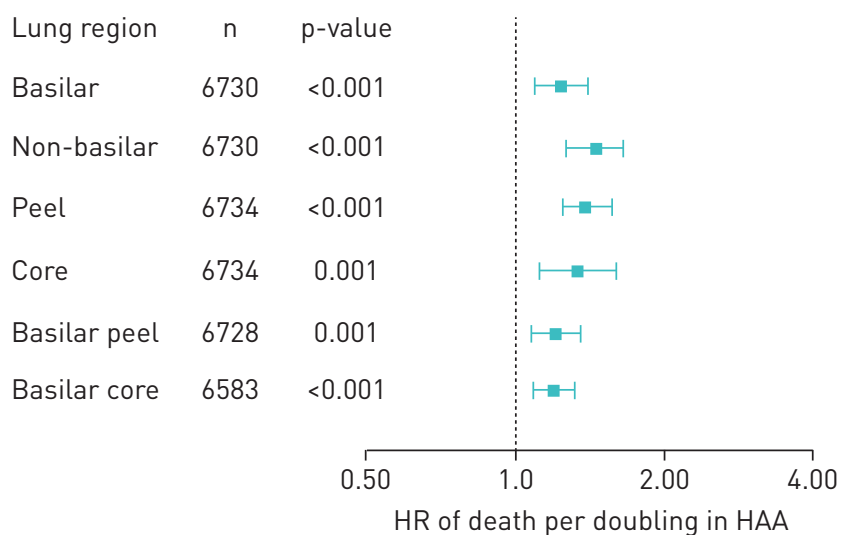

(except for FVC). This may not be surprising when considering that many of the idiopathic interstitial pneumonias have a basilar predominance [1]. Our findings provide additional support for HAA as a measure of subclinical lung injury, remodelling and inflammation. Given the consistency of our findings across the different lung regions and across multiple measures of subclinical ILD, HAA is less likely to represent significant pulmonary oedema or atelectasis, even when found within the dependent portions of the lung. Furthermore, these findings suggest that even small amounts of HAA detected in just a portion of the lung on cardiac CT have significant implications for the development of ILA and for survival.

The associations of regional HAA with MMP-7, IL-6, ILA and all-cause and ILD-specific mortality were weakest in the basilar core region. This finding may be explained by the fact that this region does not capture typical patterns of ILD found on high-resolution chest CT (HRCT). As seen in figure 1, the basilar core on the cardiac CT correlates with neither the basal nor subpleural portions of the lung where usual interstitial pneumonia (UIP) and nonspecific interstitial pneumonia (NSIP) are typically seen, nor the upper and central portions where hypersensitivity pneumonitis or sarcoidosis may be found [29-31]. The basilar core is also the region most subject to pulmonary oedema.

The associations of regional HAA with MMP-7 and FVC were strongest in the core region of the lung. This was also the only region where we found an association between HAA and SP-A. The core region captures ILDs that have a more central or airway-centred pattern on chest CT, such as hypersensitivity pneumonitis, sarcoidosis and NSIP. Parenchyma that is closer to the bronchioles is also more susceptible to inhalational exposures and aspiration injury, which may be particularly relevant in early disease in many subtypes of pulmonary fibrosis, including idiopathic pulmonary fibrosis (IPF) [14]. Our findings suggest that the core region may be involved in the earliest steps in ILD pathogenesis. The recognition of the $M U C 5 B$ variant as the strongest known risk factor for IPF has led to a new focus on the airways, where $M U C 5 B$ is expressed, in the pathogenesis of pulmonary fibrosis [32]. MUC5B overexpression has been shown to lead to impaired mucociliary clearance [33]. Others have also shown that airway abnormalities are associated with ILA and may play a role in early pulmonary fibrosis, but further mechanistic studies are still lacking [34].

On the other hand, regional HAA was most strongly associated with ILA in the basilar, peel and basilar peel portions of the lung. ILA was assessed on full lung CTs performed 10 years after the HAA measurement. ILA is a well-validated measure of subclinical ILD and represents the same visually assessed patterns seen in more advanced ILD [3-7]. Many cases of ILA represent peripheral- and basilar-predominant reticularity and ground glass opacities; more than half have a subpleural predominance [8]. It is reassuring that basilar, peel and basilar peel HAA strongly correlate with ILA. This suggests that, in many cases, the quantitative (HAA) and qualitative (ILA) assessments are measuring the same phenotype.

We found very weak (and statistically non-significant) association between FVC and HAA in the basilar, peel and basilar peel portions of the lung. This may in part reflect variation in lung volume between these regions since the basilar region has a lower lung volume than the non-basilar regions (Table S1), and there are correlations between FVC, lung volume and HAA. However, this does not explain the stronger association in the core region, which has a lower lung volume than the peel region. It is possible that, in the core region, airway collapse and resistance play bigger role in decreasing FVC and contributing to HAA. It is also possible that some of the HAA in the peripheral and basilar regions reflects dependent atelectasis, which does not contribute to reduced FVC. Interestingly, we did not find that BMI modified the association between HAA and FVC, as would be expected if artefact from obesity and compressive atelectasis was a significant contributor to HAA. 
We found that smoking was an effect modifier of the associations between HAA, MMP-7 and FVC across all lung regions [8]. Consistent with our prior work, it is likely that HAA, at least in part, reflects smoking-induced lung injury and remodelling, which can affect any lung region. Given that MMP-7 is a biomarker of lung injury, inflammation and fibrosis, it is likely elevated both in participants with subclinical ILD and in smokers [17-19]. Furthermore, as HAA is an early measure of lung injury and remodelling and can be considered a risk factor for ILD, it is likely that smoking has an additive effect on disease pathogenesis.

We found that BMI was an effect modifier of the association between HAA and MMP-7 in most lung regions, with strongest associations among overweight participants and weaker in the obese participants. Interestingly, several studies have also shown an inverse relationship between obesity and MMP-7, with downregulation of MMP-7 expression seen in obese mice [35-37]. The effect modification suggests that increased body weight may contribute to subclinical ILD and lung inflammation, but that obesity has a complex and perhaps even protective effect on subclinical ILD.

There are some limitations to our study. First, we examined both cross-sectional and longitudinal associations of exposures and outcomes collected at various time points. Thus, it is difficult to fully conclude a causative relationship amongst these measurements. Secondly, our study used cardiac CTs, which did not image the whole lung. Furthermore, these were divided into arbitrary regions that do not have anatomical correlations and may not necessarily be matched with the distribution of ILD seen in the clinical setting. Thirdly, because the MESA cohort excluded patients with underlying cardiovascular disease at baseline, the study participants were likely healthier and more ambulatory than the average population. Last, while we accounted for a number of potential confounders in our analyses, there remains the possibility of residual confounding.

\section{Conclusions}

Associations of HAA with biomarkers of lung remodelling, ILA risk, and all-cause mortality were consistent across all regions of the lung. These findings support the validity of HAA in all lung regions as a measure of subclinical ILD.

Author contributions: B. Choi drafted the initial manuscript. All authors approved the final version of the manuscript. All authors agree to be accountable for all aspects of the work.

Conflict of interest: B. Choi has nothing to disclose. S.M. Kawut reports grants from the NIH during the conduct of the study; travel support from the ATS and the Pulmonary Hypertension Association, grants for CME courses from Actelion, United Therapeutics, Gilead, Lung Biotech, Bayer and Mallinkrodt, and grants and nonfinancial support from the Cardiovascular Medical Research and Education Fund, outside the submitted work. In addition, S.M. Kawut has served in an advisory capacity (for grant review and other purposes) for United Therapeutics, Akros Pharmaceuticals, GlaxoSmithKline and Complexa Inc. without financial support or in-kind benefits. G. Raghu has nothing to disclose for this work E. Hoffman is a founder and shareholder in VIDA Diagnostics, a company commercialising lung image analysis software developed, in part, at the University of Iowa. R. Tracy has nothing to disclose. P. Madahar has nothing to disclose. E.J. Bernstein reports acting as a site PI of a clinical trial for Corbus Pharmaceuticals and grants from Pfizer, outside the submitted work. R.G. Barr reports grants from the NIH during the conduct of the study and royalties from UpToDate outside the submitted work. D.J. Lederer reports personal fees for consulting on IPF from Genentech, Pharmakea and Veracyte, personal fees for consulting on IPF and institutional funding for research from Boehringer Ingelheim and Gilead, personal fees for consulting on PF and institutional support for consultation from the Pulmonary Fibrosis Foundation, personal fees for consulting on PDF education and speaking fees from the France Foundation, and personal fees for adjudication panel membership from Degge Group, outside the submitted work. A. Podolanczuk has nothing to disclose.

Support statement: The work was funded by the National Institutes of Health contracts HHSN268201500003I and N01-HC-95159-N01-HC-95169, and grants UL1-TR-000040, UL1-TR-001079, R01-HL077612, RC1-HL100543, R01-HL-103676, K24-HL-131937, K24-HL103844, K23AR075112 and K23-HL140199. Funding information for this article has been deposited with the Crossref Funder Registry.

\section{References}

$1 \quad$ Lederer DJ, Martinez FJ. Idiopathic pulmonary fibrosis. N Engl J Med 2018; 379: 797-798.

2 King TE Jr, Pardo A, Selman M. Idiopathic pulmonary fibrosis. Lancet 2011; 378: 1949-1961.

3 Doyle TJ, Hunninghake GM, Rosas IO. Subclinical interstitial lung disease: why you should care. Am J Respir Crit Care Med 2012; 185: 1147-1153.

4 Washko GR, Hunninghake GM, Fernandez IE, et al. Lung volumes and emphysema in smokers with interstitial lung abnormalities. N Engl J Med 2011; 364: 897-906.

5 Doyle TJ, Washko GR, Fernandez IE, et al. Interstitial lung abnormalities and reduced exercise capacity. Am J Respir Crit Care Med 2012; 185: 756-762.

6 Hunninghake GM, Hatabu H, Okajima $\mathrm{Y}$, et al. MUC5B promoter polymorphism and interstitial lung abnormalities. N Engl J Med 2013; 368: 2192-2200. 
7 Putman RK, Hatabu H, Araki T, et al. Association between interstitial lung abnormalities and all-cause mortality. JAMA 2016; 315: 672-681.

8 Podolanczuk AJ, Oelsner EC, Barr RG, et al. High attenuation areas on chest computed tomography in community-dwelling adults: the MESA study. Eur Respir J 2016; 48: 1442-1452.

9 Lederer DJ, Enright PL, Kawut SM, et al. Cigarette smoking is associated with subclinical parenchymal lung disease: the Multi-Ethnic Study of Atherosclerosis (MESA)-lung study. Am J Respir Crit Care Med 2009; 180: 407-414.

10 Hartley PG, Galvin JR, Hunninghake GW, et al. High-resolution CT-derived measures of lung density are valid indexes of interstitial lung disease. J Appl Physiol 1994; 76: 271-277.

11 Podolanczuk AJ, Oelsner EC, Barr RG, et al. High-attenuation areas on chest computed tomography and clinical respiratory outcomes in community-dwelling adults. Am J Respir Crit Care Med 2017; 196: 1434-1442.

12 Kim JS, Podolanczuk AJ, Borker P, et al. Obstructive sleep apnea and subclinical interstitial lung disease in the Multi-Ethnic Study of Atherosclerosis (MESA). Ann Am Thorac Soc 2017; 14: 1786-1795.

13 Podolanczuk AJ, Raghu G, Tsai MY, et al. Cholesterol, lipoproteins and subclinical interstitial lung disease: the MESA study. Thorax 2017; 72: 472-474.

14 Leslie KO. My approach to interstitial lung disease using clinical, radiological and histopathological patterns. J Clin Pathol 2009; 62: 387-401.

15 Do KH, Lee JS, Colby TV, et al. Nonspecific interstitial pneumonia versus usual interstitial pneumonia: differences in the density histogram of high-resolution CT. J Comput Assist Tomogr 2005; 29: 544-548.

16 Mathai SK, Humphries S, Kropski JA, et al. MUC5B variant is associated with visually and quantitatively detected preclinical pulmonary fibrosis. Thorax 2019; 74: 1131-1139.

17 Rosas IO, Richards TJ, Konishi K, et al. MMP1 and MMP7 as potential peripheral blood biomarkers in idiopathic pulmonary fibrosis. PLoS Med 2008; 5: e93.

18 Richards TJ, Kaminski N, Baribaud F, et al. Peripheral blood proteins predict mortality in idiopathic pulmonary fibrosis. Am J Respir Crit Care Med 2012; 185: 67-76.

19 Zuo F, Kaminski N, Eugui E, et al. Gene expression analysis reveals matrilysin as a key regulator of pulmonary fibrosis in mice and humans. Proc Natl Acad Sci USA 2002; 99: 6292-6297.

20 Greene KE, King TE Jr., Kuroki Y, et al. Serum surfactant proteins-A and -D as biomarkers in idiopathic pulmonary fibrosis. Eur Respir J 2002; 19: 439-446.

21 Kinder BW, Brown KK, McCormack FX, et al. Serum surfactant protein-A is a strong predictor of early mortality in idiopathic pulmonary fibrosis. Chest 2009; 135: 1557-1563.

22 Kokosi MA, Margaritopoulos GA, Wells AU. Personalised medicine in interstitial lung diseases: Number 6 in the Series "Personalised medicine in respiratory diseases" Edited by Renaud Louis and Nicolas Roche. Eur Respir Rev 2018; 27: 170117.

23 Armstrong HF, Podolanczuk AJ, Barr RG, et al. Serum matrix metalloproteinase-7, respiratory symptoms, and mortality in community-dwelling adults. MESA (Multi-Ethnic Study of Atherosclerosis). Am J Respir Crit Care Med 2017; 196: 1311-1317.

24 Bild DE, Bluemke DA, Burke GL, et al. Multi-Ethnic Study of Atherosclerosis: objectives and design. Am J Epidemiol 2002; 156: 871-881.

25 Hoffman EA, Jiang R, Baumhauer H, et al. Reproducibility and validity of lung density measures from cardiac CT Scans: The Multi-Ethnic Study of Atherosclerosis (MESA) Lung Study. Acad Radiol 2009; 16: 689-699.

26 Hankinson JL, Kawut SM, Shahar E, et al. Performance of American Thoracic Society-recommended spirometry reference values in a multiethnic sample of adults: the Multi-Ethnic Study of Atherosclerosis (MESA) lung study. Chest 2010; 137: 138-145.

27 Couper D, LaVange LM, Han M, et al. Design of the Subpopulations and Intermediate Outcomes in COPD Study (SPIROMICS). Thorax 2014; 69: 491-494.

28 Hoffman EA, Ahmed FS, Baumhauer H, et al. Variation in the percent of emphysema-like lung in a healthy, nonsmoking multiethnic sample. The MESA lung study. Ann Am Thorac Soc 2014; 11: 898-907.

29 Lynch DA, Sverzellati N, Travis WD, et al. Diagnostic criteria for idiopathic pulmonary fibrosis: a Fleischner Society White Paper. Lancet Respir Med 2018; 6: 138-153.

30 Raghu G, Remy-Jardin M, Myers JL, et al. Diagnosis of idiopathic pulmonary fibrosis. An Official ATS/ERS/JRS/ ALAT Clinical Practice Guideline. Am J Respir Crit Care Med 2018; 198: e44-e68.

31 Nishino M, Itoh $\mathrm{H}$, Hatabu H. A practical approach to high-resolution CT of diffuse lung disease. Eur J Radiol 2014; 83: 6-19.

32 Seibold MA, Wise AL, Speer MC, et al. A common MUC5B promoter polymorphism and pulmonary fibrosis. N Engl J Med 2011; 364: 1503-1512.

33 Hancock LA, Hennessy CE, Solomon GM, et al. Muc5b overexpression causes mucociliary dysfunction and enhances lung fibrosis in mice. Nat Commun 2018; 9: 5363.

34 Miller ER, Putman RK, Diaz AA, et al. Increased airway wall thickness in interstitial lung abnormalities and idiopathic pulmonary fibrosis. Ann Am Thorac Soc 2019; 16: 447-454.

35 Maquoi E, Munaut C, Colige A, et al. Modulation of adipose tissue expression of murine matrix metalloproteinases and their tissue inhibitors with obesity. Diabetes 2002; 51: 1093-1101.

36 Chavey C, Mari B, Monthouel MN, et al. Matrix metalloproteinases are differentially expressed in adipose tissue during obesity and modulate adipocyte differentiation. J Biol Chem 2003; 278: 11888-11896.

37 Ress C, Tschoner A, Ciardi C, et al. Influence of significant weight loss on serum matrix metalloproteinase (MMP)-7 levels. Eur Cytokine Netw 2010; 21: 65-70. 